\title{
Prognosis of subtypes of the mucinous breast carcinoma in Chinese women: a population-based study of 32-year experience (1983-2014)
}

\author{
Bo Pan ${ }^{1, *}$, Ru Yao ${ }^{1,}{ }^{*}$, Jie Shi ${ }^{2,}$, Qian-Qian Xu' ${ }^{1}$, Yi-Dong Zhou ${ }^{1}$, Feng Mao', Yan Lin ${ }^{1}$, \\ Jing-Hong Guan ${ }^{1}$, Xue-Jing Wang ${ }^{1}$, Yan-Na Zhang ${ }^{1}$, Xiao-Hui Zhang ${ }^{1}$, Song-Jie \\ Shen $^{1}$, Ying Zhong ${ }^{1}$, Ya-Li Xu ${ }^{1}$, Qing-Li Zhu ${ }^{3}$, Zhi-Yong Liang ${ }^{2}$ and Qiang Sun ${ }^{1}$ \\ ${ }^{1}$ Department of Breast Surgery, Peking Union Medical College Hospital, Chinese Academy of Medical Sciences and Peking \\ Union Medical College, Beijing, P. R. China \\ 2 Department of Pathology, Peking Union Medical College Hospital, Chinese Academy of Medical Sciences and Peking Union \\ Medical College, Beijing, P. R. China \\ ${ }^{3}$ Department of Ultrasound, Peking Union Medical College Hospital, Chinese Academy of Medical Sciences and Peking Union \\ Medical College, Beijing, P. R. China \\ * These authors have contributed equally to this work \\ Correspondence to: Qiang Sun, email: sunqiangpumc@sina.com
}

Keywords: mucinous breast cancer, subtype, prognosis

Received: February 21, $2016 \quad$ Accepted: April 04, $2016 \quad$ Published: April 18, 2016

\section{ABSTRACT}

Purpose: The heterogeneous nature of the mucinous breast cancer (MBC), with its pure (PMBC) and mixed subtypes (MMBC), calls for precise prognosis assessment.

Methods: We analyzed 197 consecutive MBC patients, including 117 PMBC and 80 MMBC, who were treated from 1983 to 2014 . The clinicopathological features, treatment choice, disease-free survival (DFS) and overall survival (OS) were compared among PMBC, MMBC and MMBC subgroups. Prognostic factors of PMBC and MMBC were identified.

Results: Compared to PMBC, MMBC had more lymph node metastasis $(p=0.043)$, Her2 positivity $(p=0.036)$, high $\mathrm{Ki}-67$ index (defined as $>20 \%, p=0.026$ ) and antiHer2 targeted therapy $(p=0.016)$. The 5-year DFS of PMBC and MMBC were $90.4 \%$ and $86.2 \%$, whereas the 5 -year OS were $99.0 \%$ and $98.7 \%$. No significant difference was found in DFS or OS among all MBC subtypes. High Ki-67 $(p=0.020)$ appeared as DFS factor in PMBC, while anti-Her2 targeted therapy $(p=0.047)$ as the DFS predictors in MMBC.

Conclusion: MMBC manifested similar 5-year survival to PMBC in Chinese woman, suggesting that intra-tumoral heterogeneity might not interfere with MBC short-term prognosis.

\section{INTRODUCTION}

Synonymous with colloid, gelatinous mucous or mucoid carcinoma, mucinous breast cancer (MBC) represents $1-7 \%$ of all breast cancers [1-5]. The World Health Organization designates two subtypes: 1) pure mucinous breast cancer (PMBC) if the non-mucinous component is less than $10 \%$ and 2) mixed mucinous breast cancer $(\mathrm{MMBC})$ if there is $10-49 \%$ non-mucinous co-existing disease in the tumor $[6,7]$. It is generally accepted that PMBC has a favorable prognosis in both Caucasian and Chinese women compared to invasive ductal carcinomas (IDC) $[1,2]$. However, most of the studies proposing that $\mathrm{MMBC}$ had worse prognosis than PMBC were performed 2-3 decades ago, when modern adjuvant chemotherapy, radiation, endocrine and anti-Her2 
targeted therapy were largely unavailable $[3,4,8-10]$. Few studies had investigated the tumor biology, treatment choice and survival outcomes of MMBC in Chinese population, especially with respect to the intra- and intertumoral histological heterogeneity represented by the different co-existing cancer components. The prognostic predictors for PMBC and MMBC also remained unclear. A recent study showed that both the mucinous and the coexisting components in $\mathrm{MMBC}$ were remarkably similar at the molecular level to $\mathrm{PMBC}$, suggesting that MMBC be best classified as variants of mucinous cancers rather than of IDC [11]. Conversely PMBC appeared to possess phenotypic plasticity that could be converted by estrogen into MMBC with invasive lobular carcinoma (ILC) component [12]. Thus, we plan to compare the prognosis of PMBC versus MMBC in Chinese population when all measures of the modern comprehensive therapy were available.

\section{RESULTS}

\section{Descriptive information of the study cohort}

A total of 244 patients were identified as described in METHOD. After excluding 28 patients with $<50 \%$ focal mucinous lesion and 19 patients lost to follow-up, 197 patients were included in the analysis, comprising $1.9 \%$ of contemporary 10,192 breast cancer treated in PUMC Hospital. 171 patients $(86.8 \%)$ were treated during the recent ten years (2005-2014) while 130 patients $(66.0 \%)$ were treated during the recent five years (20102014). 112 patients $(56.9 \%)$ were pre-menopausal and 85 (43.1\%) post-menopausal. There were 117 PMBC and $80 \mathrm{MMBC}$ patients, the latter including 24 patients with ductal carcinoma in situ (DCIS) and IDC (with or without other types of carcinoma), 45 with only IDC, 9 with invasive micro-papillary carcinoma (IMPC) and 2 with ILC. With a median follow-up time of 41 months (3385 months), 11 PMBC and 7 MMBC patients developed recurrence or metastasis, and $1 \mathrm{PMBC}$ and $1 \mathrm{MMBC}$ passed away (Figure 1).

\section{Comparison of clinicopathological characteristics between subtypes and subgroups of $\mathrm{MBC}$}

Compared to PMBC, MMBC had significantly more lymph node metastasis $(p=0.043)$, Her2 positivity ( $p=$ 0.036 ), high Ki-67 index (defined as $>20 \%, p=0.026$ ) and anti-Her2 targeted therapy $(p=0.016)$. There were no significant differences in age at diagnosis, age group distribution, tumor size, TNM stage, ER, PR, hormone receptor status, immunophenotype, p53, type of surgery, chemotherapy, radiotherapy and endocrine therapy (Table 1). When the comparison was performed among
PMBC, MBC+DCIS+IDC, MBC+IDC and MBC+IMPC, significant differences were identified in lymph node metastasis $(p=0.023)$, Her2 positivity $(p=0.014)$, high Ki-67 index $(p=0.008)$, chemotherapy $(p=0.011)$ and anti-Her2 targeted therapy $(p=0.002)$ (Table 2$)$.

\section{Survival outcomes and prognostic factors of MBC subtypes}

The 5-year DFS of PMBC and MMBC were 90.4\% and $86.2 \%$, whereas the 5 -year OS were $99.0 \%$ and $98.7 \%$ respectively. The 5 -year DFS and OS for MMBC subgroups were: $85.7 \%$ and $100.0 \%$ for MBC+DCIS+IDC, $83.5 \%$ and $97.6 \%$ for $\mathrm{MBC}+\mathrm{IDC}$, and $100.0 \%$ and $100.0 \%$ for MBC+IMPC. No significant difference was found in DFS or OS either between PMBC vs MMBC or among the above mentioned MMBC subgroups (Figure 2, Table $3,4)$. High Ki-67 index (defined as $>20 \%, p=0.020$ ) was identified as the significant DFS prognostic factor for PMBC, whereas anti-Her2 targeted therapy $(p=0.047)$ appeared to be the DFS predictor for MMBC (Table 5, 6). DFS stratified by Ki-67 in PMBC and by anti-Her2 targeted therapy in MMBC both showed significant differences (Figure 2). ER, PR, hormone receptor status, immunophenotype and endocrine therapy might be potential DFS predictors according to univariate analysis. However, these factors were not significant in the multivariate analysis. None of the clinicopathological and treatment factors listed above could serve as OS predictors for MBC subtypes due to the limited OS events.

\section{DISCUSSION}

$\mathrm{MBC}$ is one of the most commonly seen special types of breast cancer $[1,2,4,8]$. Experience in diagnosis and treatment of $\mathrm{MBC}$ was usually acquired from retrospective studies instead of prospective randomized trials. It was widely believed that $\mathrm{MMBC}$ had a poorer prognosis than PMBC [3, 4, 8-10]. However, these retrospective studies were mainly based on data from Caucasian, and mostly performed during the 1960 s to 1980s, when anti-Her2 targeted therapy, most of the endocrine therapy, chemotherapy and radiation therapy were unavailable. Thus the poorer outcome of MMBC might be due to insufficient treatment. Additionally, MMBC is not a single disease. Whether MMBC subgroups have different survival outcomes remains unclear. A recent study reported differences in breast cancer epidemiology, clinical characteristics and prognosis between Chinese and Caucasian women [13, 14]. However, few studies have evaluated the survival outcome among MBC subtypes in Chinese women, who tend to develop breast cancer and $\mathrm{MBC}$ at a much younger age than Caucasian counterparts $[1,2,5,15,16]$.

Although PMBC usually had normal diploid DNA 
Table 1: Clinicopathological characteristics of PMBC and MMBC patients

\begin{tabular}{|c|c|c|c|}
\hline \multirow{2}{*}{ Characteristics } & \multicolumn{2}{|c|}{ No. (\%) of Patients } & \multirow{2}{*}{$P^{a}$} \\
\hline & PMBC & ММВC & \\
\hline Total & 117 & 80 & \\
\hline Age (Mean \pm SD) (years) & $53.26 \pm 15.25$ & $55.90 \pm 14.38$ & 0.223 \\
\hline Age at diagnosis (years) & & & 0.432 \\
\hline$\leq 35$ & $10(8.5)$ & $5(6.2)$ & \\
\hline $36 \sim 50$ & $52(44.5)$ & $30(37.5)$ & \\
\hline$>50$ & $55(47.0)$ & $45(56.3)$ & \\
\hline Tumor size (cm) & & & 0.480 \\
\hline $\mathrm{T} \leq 2.0$ & $59(50.4)$ & $43(53.8)$ & \\
\hline $2<\mathrm{T} \leq 5.0$ & $49(41.9)$ & $31(38.8)$ & \\
\hline $\mathrm{T}>5.0$ & $4(3.4)$ & $5(6.2)$ & \\
\hline Unknown & $5(4.3)$ & $1(1.2)$ & \\
\hline Lymph node status & & & 0.043 \\
\hline Negative & $103(88.0)$ & $64(80.0)$ & \\
\hline Positive & $11(9.4)$ & $16(20.0)$ & \\
\hline Unknown & $3(2.6)$ & $0(0.0)$ & \\
\hline TNM stage s $^{b}$ & & & 0.147 \\
\hline Stage I & $55(47.0)$ & $35(43.8)$ & \\
\hline Stage II & $53(45.3)$ & $35(43.8)$ & \\
\hline Stage III & $6(5.1)$ & $10(12.5)$ & \\
\hline Unknown & $3(2.6)$ & $0(0.0)$ & \\
\hline ER status & & & 0.484 \\
\hline Positive & $94(80.3)$ & $66(82.5)$ & \\
\hline Negative & $12(10.3)$ & $10(12.5)$ & \\
\hline Unknwon & $11(9.4)$ & $4(5.0)$ & \\
\hline PR status & & & 0.834 \\
\hline Positive & $88(75.3)$ & $62(77.6)$ & \\
\hline Negative & $19(16.2)$ & $13(16.2)$ & \\
\hline Unknwon & $10(8.5)$ & $5(6.2)$ & \\
\hline Hormone receptor status & & & 0.631 \\
\hline Positive & $98(83.8)$ & $70(87.5)$ & \\
\hline Negative & $9(7.7)$ & $6(7.5)$ & \\
\hline Unknwon & $10(8.5)$ & $4(5.0)$ & \\
\hline
\end{tabular}




\begin{tabular}{|c|c|c|c|}
\hline HER2 status & & & 0.036 \\
\hline Positive & $3(2.6)$ & $9(11.2)$ & \\
\hline Negative & 97 (82.9) & $58(72.5)$ & \\
\hline Unknwon & $17(14.5)$ & $13(16.2)$ & \\
\hline Ki-67 expression & & & 0.026 \\
\hline$<20 \%$ & $64(54.7)$ & $36(45.0)$ & \\
\hline$\geq 20 \%$ & $29(24.8)$ & $34(42.5)$ & \\
\hline Unknown & $24(20.5)$ & $10(12.5)$ & \\
\hline Immunophenotype & & & 0.136 \\
\hline Luminal A & $62(53.0)$ & $32(40.0)$ & \\
\hline Luminal B & $29(24.8)$ & $32(40.0)$ & \\
\hline HER2 & $0(0.0)$ & $1(1.2)$ & \\
\hline TNBC & $8(6.8)$ & $5(6.2)$ & \\
\hline Unknown & $18(15.4)$ & $10(12.5)$ & \\
\hline p53 & & & 0.547 \\
\hline Positive & $6(5.1)$ & $6(7.5)$ & \\
\hline Negative & $46(39.3)$ & $26(32.5)$ & \\
\hline Unknown & 65 (55.6) & $48(60.0)$ & \\
\hline Surgery & & & 0.897 \\
\hline Mastectomy & $75(64.1)$ & $52(65.0)$ & \\
\hline Breast conserving & $42(35.9)$ & $28(35.0)$ & \\
\hline Unknown & $0(0.0)$ & $0(0.0)$ & \\
\hline Chemotherapy & & & 0.195 \\
\hline No & $69(59.0)$ & $41(51.2)$ & \\
\hline Yes & $43(36.8)$ & $38(47.5)$ & \\
\hline Unknown & $5(4.2)$ & $1(1.2)$ & \\
\hline Radiotherapy & & & 0.242 \\
\hline No & $82(70.1)$ & $57(71.3)$ & \\
\hline Yes & $26(22.2)$ & $21(20.2)$ & \\
\hline Unknown & $9(7.7)$ & $2(2.5)$ & \\
\hline Anti-Her2 targeted therapy & & & 0.016 \\
\hline No & $107(91.5)$ & $70(87.5)$ & \\
\hline Yes & $2(1.7)$ & $8(10.0)$ & \\
\hline
\end{tabular}




\begin{tabular}{|l|l|l|l|}
\hline Unknown & $8(6.8)$ & $2(2.5)$ & \\
\hline Endocrine therapy & & & 0.399 \\
\hline No & $15(12.8)$ & $8(10.0)$ & \\
\hline Yes & $91(77.8)$ & $68(85.0)$ & \\
\hline Unknown & $11(9.4)$ & $4(5.0)$ & \\
\hline
\end{tabular}

Abbreviations: PMBC, pure mucinous breast cancer; MBC, mucinous breast cancer; SD, standard deviation; TNM, tumor, node, metastasis system; ER, estrogen receptor; PR, progesterone receptor; TNBC, triple negative breast cancer.

a Bold type indicates statistical significance.

${ }^{\mathrm{b}}$ TNM stage is according to the $7^{\text {th }}$ AJCC cancer staging system.

stemline whereas MMBC harbored aneuploid DNA content, a recent study suggested that MBC subtypes based on gene expression profiling might be more complex than anticipated $[17,18]$. Unsupervised clustering analysis showed that MMBC displayed similar patterns of genetic aberrations and preferentially clustered together with

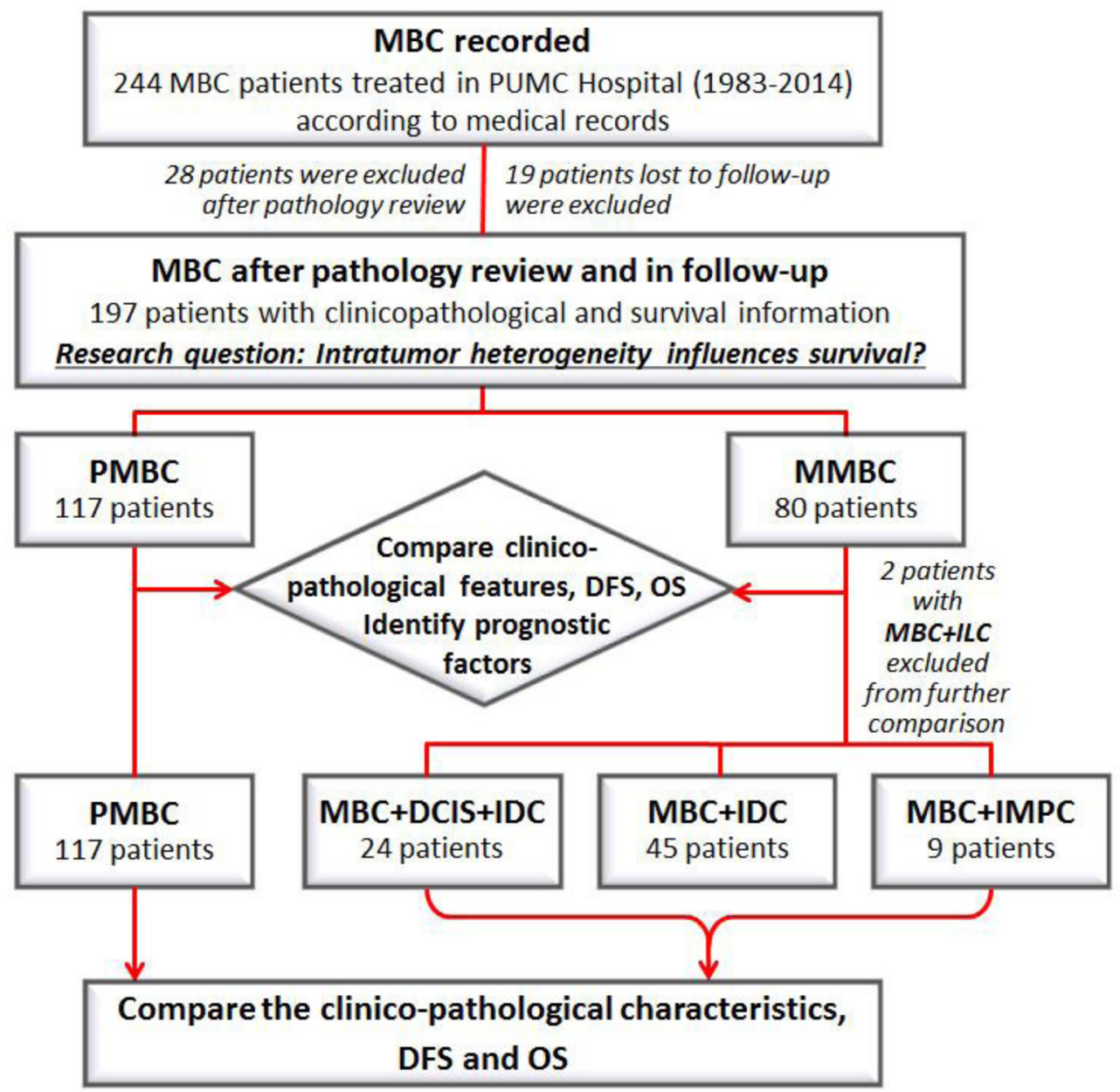

Figure 1: Diagram of the research design. The clinic-pathological characteristics and the survival outcomes (DFS and OS) were firstly compared between PMBC and MMBC, and then between PMBC, MBC+DCIS+IDC, MBC+IDC and MBC+IMPC. Two patients with $\mathrm{MBC}+\mathrm{ILC}$ were excluded from the second comparison due to limited case number. Abbreviations: MBC, mucinous breast cancer; PMBC, pure mucinous breast cancer; MMBC, mixed mucinous breast cancer; DCIS, ductal carcinoma in situ; IDC, invasive ductal carcinoma; ILC, invasive lobular carcinoma; IMPC, invasive micropapillary carcinoma; DFS, disease free survival; OS, overall survival. 

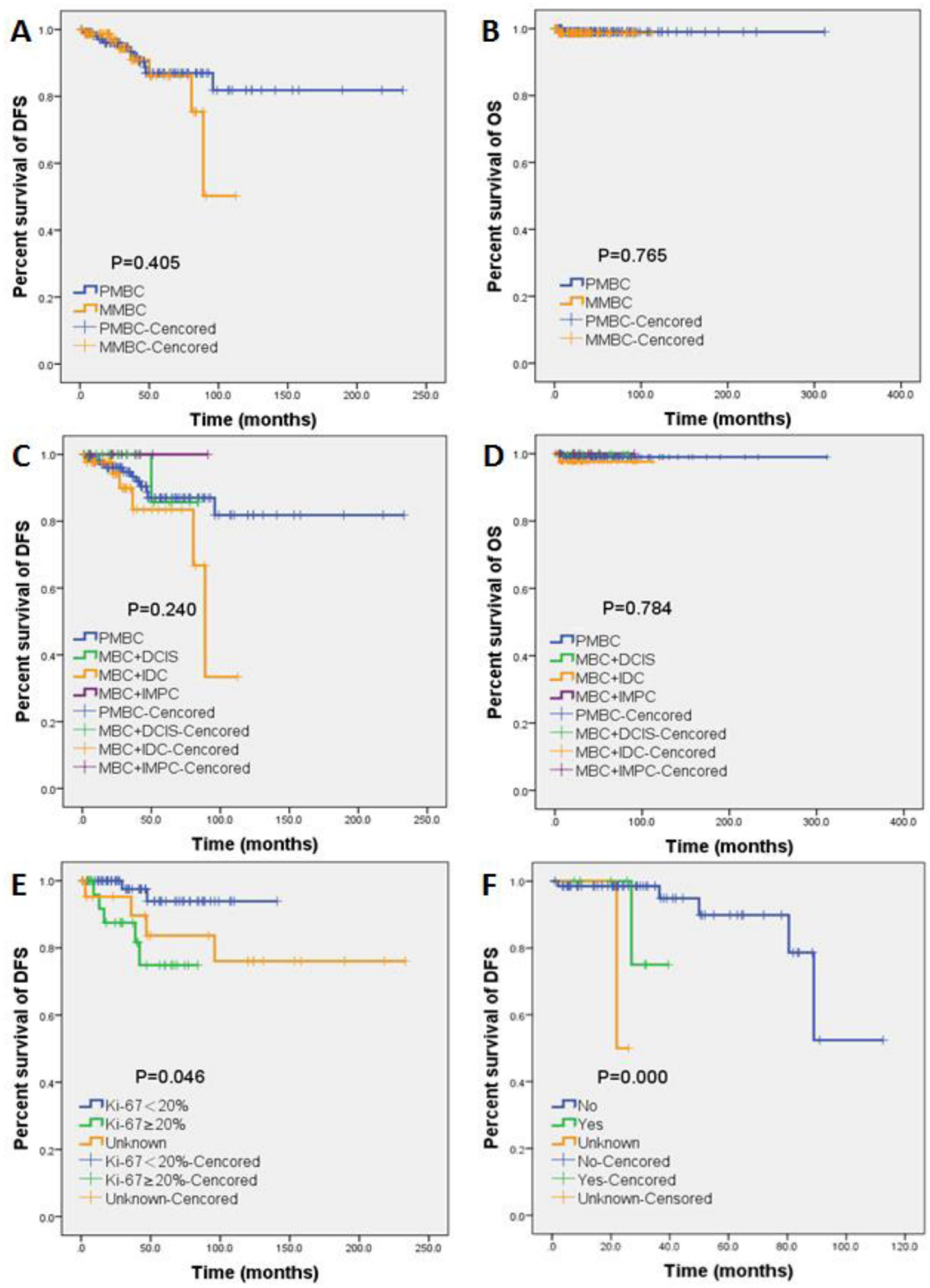

Figure 2: Kaplan-Meier estimates of DFS and OS of MBC patients. DFS A. and OS B. compared between PMBC and MMBC patients. The comparison of DFS C. and OS D. of PMBC, MBC+DCIS+IDC, MBC+IDC, and MBC+IMPC patients. DFS of PMBC patients compared between subgroups of Ki-67 high (defined as $\geq 20 \%$ ) versus Ki-67 low (defined as $<20 \%$ ) E. DFS of MMBC patients compared between patient subgroups with or without anti-Her2 targeted therapy $\mathbf{F}$. 
Table 2: Comparison of the clinicopathological characteristics of $\mathrm{PMBC}$ versus subgroups of $\mathrm{MMBC}$ including $\mathrm{MBC}+\mathrm{DCIS}+\mathrm{IDC}, \mathrm{MBC}+\mathrm{IDC}$ and MBC+IMPC patients

\begin{tabular}{|c|c|c|c|c|c|}
\hline \multirow{2}{*}{ Characteristics } & \multicolumn{4}{|c|}{ No. (\%) of Patients } & \multirow{2}{*}{$P^{a}$} \\
\hline & PMBC & MBC+DCIS+IDC & MBC+IDC & MBC+IMPC & \\
\hline Total & 117 & 24 & 45 & 9 & \\
\hline Age (Mean \pm SD) (years) & $53.3 \pm 15.3$ & $56.6 \pm 12.6$ & $54.7 \pm 15.2$ & $61.8 \pm 15.4$ & 0.333 \\
\hline Age at diagnosis (years) & & & & & 0.612 \\
\hline$\leq 35$ & $10(8.5)$ & $0(0.0)$ & $4(8.9)$ & $1(11.1)$ & \\
\hline $36 \sim 50$ & $52(44.5)$ & $10(41.7)$ & $17(37.8)$ & $2(22.2)$ & \\
\hline$>50$ & $55(47.0)$ & $14(58.3)$ & $24(53.3)$ & $6(66.7)$ & \\
\hline Tumor size (cm) & & & & & 0.204 \\
\hline $\mathrm{T} \leq 2.0$ & $59(50.4)$ & $18(75.0)$ & $19(42.2)$ & $5(56.6)$ & \\
\hline $2<\mathrm{T} \leq 5.0$ & 49 (41.9) & $4(16.7)$ & $24(53.3)$ & $3(33.3)$ & \\
\hline $\mathrm{T}>5.0$ & $4(3.4)$ & $1(4.2)$ & $2(4.4)$ & $1(11.1)$ & \\
\hline Unknown & $5(4.3)$ & $1(4.2)$ & $0(0.0)$ & $0(0.0)$ & \\
\hline Lymph node status & & & & & 0.023 \\
\hline Negative & $103(88.0)$ & $22(91.7)$ & $32(71.1)$ & $9(100.0)$ & \\
\hline Positive & $11(9.4)$ & $2(8.3)$ & $13(28.9)$ & $0(0.0)$ & \\
\hline Unknown & $3(2.6)$ & $0(0.0)$ & $0(0.0)$ & $0(0.0)$ & \\
\hline TNM stage $^{\mathrm{b}}$ & & & & & 0.069 \\
\hline Stage I & $55(47.0)$ & $16(66.7)$ & $13(28.9)$ & $5(55.6)$ & \\
\hline Stage II & $53(45.3)$ & $6(25.0)$ & $25(55.6)$ & $4(44.4)$ & \\
\hline Stage III & $6(5.1)$ & $2(8.3)$ & $7(15.6)$ & $0(0.0)$ & \\
\hline Unknown & $3(2.6)$ & $0(0.0)$ & $0(0.0)$ & $0(0.0)$ & \\
\hline ER status & & & & & 0.587 \\
\hline Positive & $94(80.3)$ & $22(91.7)$ & $34(75.6)$ & $8(88.9)$ & \\
\hline Negative & $12(10.3)$ & $2(8.3)$ & $7(15.6)$ & $1(11.1)$ & \\
\hline Unknown & $11(9.4)$ & $0(0.0)$ & $4(8.9)$ & $0(0.0)$ & \\
\hline PR status & & & & & 0.816 \\
\hline Positive & $88(75.2)$ & $21(87.5)$ & $33(73.3)$ & $7(77.8)$ & \\
\hline Negative & $19(16.2)$ & $2(8.3)$ & $8(17.8)$ & $2(22.2)$ & \\
\hline Unknown & $10(8.5)$ & $1(4.2)$ & $4(8.9)$ & $0(0.0)$ & \\
\hline Hormone receptor status & & & & & 0.694 \\
\hline Positive & $98(83.8)$ & $23(95.8)$ & $37(82.2)$ & $8(88.9)$ & \\
\hline Negative & $9(7.7)$ & $1(4.2)$ & $4(8.9)$ & $1(11.1)$ & \\
\hline Unknown & $10(8.5)$ & $0(0.0)$ & $4(8.9)$ & $0(0.0)$ & \\
\hline HER2 status & & & & & 0.014 \\
\hline
\end{tabular}




\begin{tabular}{|c|c|c|c|c|c|}
\hline Positive & $3(2.6)$ & $1(4.2)$ & $8(17.8)$ & $0(0.0)$ & \\
\hline Negative & $97(82.9)$ & $20(83.3)$ & $28(62.2)$ & $8(88.9)$ & \\
\hline Unknown & $17(14.5)$ & $3(12.5)$ & $9(20.0)$ & $1(11.1)$ & \\
\hline Ki-67 expression & & & & & 0.008 \\
\hline$<20 \%$ & $64(54.7)$ & $16(66.7)$ & $14(31.1)$ & $6(66.7)$ & \\
\hline$\geq 20 \%$ & $29(24.8)$ & $5(20.8)$ & $24(53.3)$ & $3(33.3)$ & \\
\hline Unknown & $24(20.5)$ & $3(12.5)$ & $7(15.6)$ & $0(0.0)$ & \\
\hline Immunophenotype & & & & & 0.105 \\
\hline Luminal A & $62(53.0)$ & $15(62.5)$ & $12(26.7)$ & $5(55.6)$ & \\
\hline Luminal B & $29(24.8)$ & $5(20.8)$ & $22(48.9)$ & $3(33.3)$ & \\
\hline HER2 & $0(0.0)$ & $0(0.0)$ & $1(2.2)$ & $0(0.0)$ & \\
\hline TNBC & $8(6.8)$ & $1(4.2)$ & $3(6.7)$ & $1(11.1)$ & \\
\hline Unknown & $18(15.4)$ & $3(12.5)$ & $7(15.6)$ & $0(0.0)$ & \\
\hline p53 & & & & & 0.418 \\
\hline Positive & $6(5.1)$ & $0(0.0)$ & $5(11.1)$ & $1(11.1)$ & \\
\hline Negative & $46(39.3)$ & $7(29.2)$ & $16(35.6)$ & $2(22.2)$ & \\
\hline Unknown & 65 (55.6) & $17(70.8)$ & $24(53.3)$ & $6(66.7)$ & \\
\hline Surgery & & & & & 0.575 \\
\hline Mastectomy & $75(64.1)$ & $15(62.5)$ & $31(68.9)$ & $4(44.4)$ & \\
\hline Breast conserving & $42(35.9)$ & $9(37.5)$ & $14(31.1)$ & $5(55.6)$ & \\
\hline Unknown & $0(0.0)$ & $0(0.0)$ & $0(0.0)$ & $0(0.0)$ & \\
\hline Chemotherapy & & & & & 0.011 \\
\hline No & $69(59.0)$ & $18(75.0)$ & $16(35.6)$ & $6(66.7)$ & \\
\hline Yes & $43(36.8)$ & $5(20.8)$ & 29 (64.4) & $3(33.3)$ & \\
\hline Unknown & $5(4.2)$ & $1(4.2)$ & $0(0.0)$ & $0(0.0)$ & \\
\hline Radiotherapy & & & & & 0.299 \\
\hline No & $82(70.1)$ & $19(79.2)$ & $30(66.7)$ & $6(66.7)$ & \\
\hline Yes & $26(22.2)$ & $4(16.7)$ & $15(33.3)$ & $3(33.3)$ & \\
\hline Unknown & $9(7.7)$ & $1(4.1)$ & $0(0.0)$ & $0(0.0)$ & \\
\hline Anti-Her2 targeted therapy & & & & & 0.002 \\
\hline No & 107 (91.5) & $23(95.8)$ & $36(80.0)$ & $9(100.0)$ & \\
\hline Yes & $2(1.7)$ & $0(0.0)$ & $8(17.8)$ & $0(0.0)$ & \\
\hline Unknown & $8(6.8)$ & $1(4.1)$ & $1(2.2)$ & $0(0.0)$ & \\
\hline Endocrine therapy & & & & & 0.566 \\
\hline No & $15(12.8)$ & $1(4.2)$ & $6(13.3)$ & $1(11.1)$ & \\
\hline Yes & $91(77.8)$ & $21(87.5)$ & $38(84.4)$ & $8(88.9)$ & \\
\hline Unknown & $11(9.4)$ & $2(8.3)$ & $1(2.2)$ & $0(0.0)$ & \\
\hline
\end{tabular}

Abbreviations: $\mathrm{PMBC}$, pure mucinous breast cancer; $\mathrm{MBC}$, mucinous breast cancer; DCIS, ductal carcinoma in situ; IDC, invasive ductal carcinoma; IMPC, invasive micropapillary carcinoma; SD, standard deviation; TNM, tumor, node, metastasis system; ER, estrogen receptor; PR, progesterone receptor.

${ }^{a}$ Bold type indicates statistical significance.

${ }^{\mathrm{b}} \mathrm{TNM}$ stage is according to the $7^{\mathrm{th}} \mathrm{AJCC}$ cancer staging system. 
Table 3: Kaplan-Meier estimated DFS and OS rates compared between PMBC and MMBC

\begin{tabular}{|l|l|l|l|l|l|}
\hline Group & No. of patients & 5-year DFS (\%) & P & 5-year OS (\%) & P \\
\hline PMBC & 117 & 90.4 & \multirow{2}{*}{0.405} & 99.0 & \multirow{2}{*}{0.765} \\
\cline { 1 - 1 } & 80 & 86.2 & & 98.7 & \\
\hline
\end{tabular}

Abbreviations: PMBC, pure mucinous breast cancer; MMBC, mixed mucinous breast cancer; DFS, disease free survival; OS, overall survival.

Table 4: Kaplan-Meier estimated DFS and OS rates compared between PMBC, MBC+DCIS+IDC, MBC+IDC and MBC+IMPC

\begin{tabular}{|c|c|c|c|c|c|}
\hline Group & No. of patients & 5-year DFS (\%) & $P$ & 5-year OS (\%) & $P$ \\
\hline PMBC & 117 & 90.4 & \multirow{4}{*}{0.240} & 99.0 & \multirow{4}{*}{0.784} \\
\hline $\mathrm{MBC}+\mathrm{DCIS}+\mathrm{IDC}$ & 24 & 85.7 & & 100.0 & \\
\hline $\mathrm{MBC}+\mathrm{IDC}$ & 45 & 83.5 & & 97.6 & \\
\hline $\mathrm{MBC}+\mathrm{IMPC}$ & 9 & 100.0 & & 100.0 & \\
\hline
\end{tabular}

Abbreviations: $\mathrm{MBC}$, mucinous breast cancer; $\mathrm{PMBC}$, pure mucinous breast cancer; $\mathrm{MMBC}$, mixed mucinous breast cancer; DCIS, ductal carcinoma in situ; IDC, invasive ductal carcinoma; IMPC, invasive micropapillary carcinoma; DFS, disease free survival; OS, overall survival.

Table 5: Univariate and multivariate Cox analysis of disease-free survival of patients with PMBC

\begin{tabular}{|l|l|l|l|}
\hline \multirow{2}{*}{ Variables } & Univariate $^{\mathbf{a}}$ & Multivariate $^{\mathbf{b}}$ & \multicolumn{2}{l|}{} \\
\cline { 2 - 4 } & $\boldsymbol{P}^{\mathbf{c}}$ & HR (95\% CI) & $\boldsymbol{P}^{\mathbf{c}}$ \\
\hline Age at diagnosis & 0.170 & $0.258(0.056-1.186)$ & 0.082 \\
\hline Tumor size & 0.415 & $0.358(0.023-5.662)$ & 0.466 \\
\hline Lymph node status & 0.331 & $5.666(0.358-89.609)$ & 0.218 \\
\hline TNM staged & 0.831 & $2.546(0.083-78.191)$ & 0.593 \\
\hline ER status & 0.697 & $1512.053(0-9.453 \mathrm{E}+138)$ & 0.963 \\
\hline PR status & 0.496 & $0.247(0.028-2.203)$ & 0.210 \\
\hline Hormone receptor status & 0.741 & $0.004(0-2.834 \mathrm{E}+133)$ & 0.973 \\
\hline HER2 status & 0.631 & $1.796(0.359-8.988)$ & 0.476 \\
\hline Ki-67 expression & $\mathbf{0 . 0 4 6}$ & $\mathbf{5 8 . 7 2 2}(\mathbf{1 . 8 8 9}-1825.766)$ & $\mathbf{0 . 0 2 0}$ \\
\hline Immunophenotype & 0.111 & $0.169(0.025-1.135)$ & 0.067 \\
\hline p53 & 0.801 & $1.857(0.676-5.106)$ & 0.230 \\
\hline Surgery & 0.054 & $0.077(0.005-1.227)$ & 0.070 \\
\hline Chemotherapy & 0.379 & $0.420(0.070-2.517)$ & 0.082 \\
\hline Radiotherapy & 0.738 & $1.051(0.095-11.576)$ & 0.070 \\
\hline Anti-Her2 targeted therapy & 0.874 & $0.078(0.004-1.629)$ & 0.466 \\
\hline Endocrine therapy & 0.945 & $8.401(0.402-175.734)$ & 0.218 \\
\hline
\end{tabular}

Abbreviations: PMBC, pure mucinous breast cancer; ER, estrogen receptor; PR, progesterone receptor.

${ }^{a}$ Kaplan-Meier univariate analysis including all factors.

${ }^{\mathrm{b}}$ Adjusted by Cox proportional hazard regression model including all factors with method of enter.

${ }^{\mathrm{c}}$ Bold type indicates statistical significance.

d TNM stage is according to the $7^{\text {th }}$ AJCC cancer staging system.

PMBC rather than with IDC [11]. A study with MBC cell line and xenograft model also showed that PMBC manifested phenotypic plasticity and could be converted by estrogen into MMBC with ILC [12]. This genotypic and phenotypic similarity between $\mathrm{PMBC}$ and MMBC provides explanation for their similar prognosis. Secretory mucins (MUC2 and MUC6) and the mucus might also act as a barrier to cancerous extension and decrease the aggressiveness of the tumor biology $[8,19]$.
In our study, the difference in lymph node (LN) metastasis between PMBC, MMBC and MMBC subtypes might be due to distinct tumor biology. However, the MBC were diagnosed at similar $\mathrm{T}$ stage and hence have no significant differences in pTNM stage. Our result on MBC survival coincided with the study from Park $S$ et al. reporting similar 10-year DFS and OS between PMBC and MMBC [20]. Bae SY et al. reported similar DFS and different OS $(p=0.043)$, however, their study did not 
Table 6: Univariate and multivariate Cox analysis of disease-free survival of patients with MMBC

\begin{tabular}{|l|l|l|l|}
\hline \multirow{2}{*}{ Variables } & Univariate $^{\mathbf{a}}$ & Multivariate $^{\mathbf{b}}$ & \\
\cline { 2 - 4 } & $\mathbf{P}^{\mathbf{c}}$ & HR (95\% CI) & $\boldsymbol{P}^{\mathbf{c}}$ \\
\hline Pathologic types & 0.460 & $0.344(0.015-8.149)$ & 0.509 \\
\hline Age at diagnosis & 0.606 & $1.083(0.048-24.428$ & 0.960 \\
\hline Tumor size & 0.764 & $0.052(0.000-31.232)$ & 0.364 \\
\hline Lymph node status & 0.573 & $0.000(0.000-355.483)$ & 0.145 \\
\hline TNM staged & 0.618 & $3154(0.032-3126)$ & 0.170 \\
\hline ER status & $\mathbf{0 . 0 0 0}$ & $0.004(0.000-5.52)$ & 0.136 \\
\hline PR status & $\mathbf{0 . 0 0 5}$ & $3.696(0.039-347.957)$ & 0.573 \\
\hline Hormone receptor status & $\mathbf{0 . 0 0 0}$ & $3.246(0.004-2777)$ & 0.733 \\
\hline HER2 status & 0.504 & $0.092(0.002-3.521)$ & 0.199 \\
\hline Ki-67 expression & 0.302 & $12.349(0.005-33822)$ & 0.534 \\
\hline Immunophenotype & $\mathbf{0 . 0 0 0}$ & $1.055(0.055-20.270)$ & 0.971 \\
\hline p53 & 0.067 & $2.025(0.086-47.626)$ & 0.662 \\
\hline Surgery & 0.962 & $0.025(0.000-176.301)$ & 0.414 \\
\hline Chemotherapy & 0.232 & $0.172(0.001-23.738)$ & 0.483 \\
\hline Radiotherapy & 0.473 & $27.030(0.012-60963)$ & 0.403 \\
\hline Anti-Her2 targeted therapy & $\mathbf{0 . 0 0 0}$ & $\mathbf{6 9 7 7}(\mathbf{1 . 1 4 1 0}-\mathbf{4 2 6 9 6 0 7 9 )}$ & $\mathbf{0 . 0 4 7}$ \\
\hline Endocrine therapy & $\mathbf{0 . 0 0 3}$ & $0.071(0.001-3.380)$ & 0.180 \\
\hline
\end{tabular}

Abbreviations: MMBC, mixed mucinous breast cancer; ER, estrogen receptor; PR, progesterone receptor.

${ }^{\text {a }}$ Kaplan-Meier univariate analysis including all factors.

${ }^{\mathrm{b}}$ Adjusted by Cox proportional hazard regression model including all factors with method of enter.

${ }^{\mathrm{c}}$ Bold type indicates statistical significance.

d TNM stage is according to the $7^{\text {th }}$ AJCC cancer staging system.

review pTNM stage, Ki-67, anti-Her2 targeted therapy [21]. Additionally, the age at diagnosis of MBC patients was much younger than contemporary IDC in Korean women, which was different from both Caucasian and Chinese $[1,2,5,20,21]$. Zhang $\mathrm{M}$ et al. reported better PMBC survival than MMBC in Chinese population [5]. However, most of the MMBC patients included in that study were diagnosed at a much later stage than PMBC, while $70.5 \%$ of $\mathrm{PMBC}$ patients received chemotherapy. There was no data concerning the Her2 status so that the similar percentage of anti-Her2 targeted therapy between MBC vs non-MBC or between PMBC vs MMBC would be difficult to interpret.

Compared with IDC, IMPC usually has larger size, more metastatic lymph nodes, increased lymphovascular invasion (LVI) and more aggressive behavior [22]. Poorer survival was also observed for breast carcinoma containing IMPC component [22]. Notably, a special subtype of PMBC with micropapillary epithelial growth pattern was identified as invasive micropapillary mucinous carcinoma (IMPMC) [23], or mucinous carcinomas with a micropapillary pattern (MUMPC) [24]. This heterogeneous PMBC had more LN metastasis, higher Her2 expression, LVI, and a poorer prognosis than pure PMBC [23, 24]. Meanwhile, it showed decreased LN metastasis, lower nuclear grade, higher expression of ER and PR, less expression of Her2, and better prognosis compared to IMPC. Though controversial, it was proposed that PMBC, MUMPC/IMPMC and IMPC might represent clinical entities within a spectrum of heterogeneous diseases, with different percentage of mucin secretion and micropapillary components [23-25]. The MBC+IMPC in our study was different from MUMPC/IMPMC and did not exhibit higher LN metastasis, higher Her2 or Ki-67 expression, or poorer survival outcome.

Our study had several limitations. Firstly, it was a single-center study with limited case number, and two patients with MBC+ILC had to be excluded from the comparison. Secondly, although this retrospective study reviewed MBC patients distributed during 32 years' time span, majority $(86.8 \%)$ of patients was treated in the recent decade (2005-2014), so it would make more sense to analyze the 5 -year short-term survival. There might still be significant difference in long-term 10-year prognosis between PMBC and MMBC, because MBC is basically luminal subtype and have shown late recurrences after 10 years $[24,26]$. Thirdly, LN metastasis was not identified as the DFS predictor in our study, although it was identified in other studies to be candidate prognostic factor for PMBC [1, 2, 8, 21, 26, 27]. Fourthly, Ki-67 expression was only documented in $79.5 \%$ of the PMBC and $87.5 \%$ of the MMBC, while p53 status in more than half of the cases was unknown.

In conclusion, our study revealed that $\mathrm{MMBC}$ had similar short-term survival as PMBC in Chinese patients, suggesting that intra-tumoral heterogeneity might not 
interfere with MBC prognosis in Chinese woman. Ki-67 proliferation index was identified as a DFS prognostic factor for PMBC, whereas anti-Her2 targeted therapy as the potential DFS predictor for MMBC. Further studies with increased cases number, prolonged follow-up and improved bio-markers need to be performed to gain a deeper understanding of MBC biology and prognosis with respect to intra- and inter-tumoral heterogeneity.

\section{MATERIALS AND METHODS}

\section{Ethics statement}

This study was approved by the Ethics Committee of the Peking Union Medical College Hospital, Chinese Academy of Medical Sciences.

\section{Patient selection, pathology review and follow-up}

From January 1983 to December 2014, 244 consecutive MBC patients were treated primarily with breast cancer surgeries in PUMC Hospital according to the medical records searching. All patients' formalin-fixed paraffin-embedded (FFPE) pathological sections were reviewed and 28 patients with focal mucinous components $<50 \%$ of the total cancerous lesions were excluded from the study. All patients were followed by telephone call, by out-patient clinics records of follow-up examinations or by both measures. Another 19 patients who were lost to follow-up were also excluded.

There were $197 \mathrm{MBC}$ patients, including 117 PMBC and $80 \mathrm{MMBC}$, in the study cohort. The clinicopathological characteristics, treatment choice, DFS and OS were compared both between 117 PMBC vs 80 MMBC, and among all MBC subgroups, including 24 MMBC with DCIS and IDC (with or without other types of carcinoma), 45 with only IDC and 9 with IMPC. Two patients with MMBC and ILC were excluded from the comparison due to the small case number. DFS factors of $\mathrm{PMBC}$ and $\mathrm{MMBC}$ were identified respectively. Identification of prognostic factors for MMBC subgroups were not performed also due to the limited case numbers (Figure 1).

\section{Statistical analysis}

The quantitative variables were compared with $t$-test and the categorical variables were compared with chi-square tests. Survival outcomes including 5-year predicted DFS and OS were analyzed and compared by the Kaplan-Meier curve method. Kaplan-Meier univariate analyses and Cox multivariate analyses were performed to identify the prognostic factors for PMBC and MMBC respectively. The significance threshold was set at $p<$ 0.05. SPSS software, version 18.0 (SPSS, Inc. Chicago, IL, US) was used for all of the statistical analyses.

\section{ACKNOWLEDGMENTS}

We would like to thank all of the patients for their participation in this study.

\section{FUNDING SUPPORT}

This work was supported by the Twelfth Five Year Key Programs for Science and Technology Development of China (Grant No. 2014BAI028B03) and the Natural Science Foundation of China (Grant No. 81001183).

\section{CONFLICTS OF INTEREST}

The authors have declared that no competing interests exist.

\section{REFERENCES}

1. Di Saverio S, Gutierrez J and Avisar E. A retrospective review with long term follow up of 11,400 cases of pure mucinous breast carcinoma. Breast cancer research and treatment. 2008; 111:541-547.

2. Cao AY, He M, Liu ZB, Di GH, Wu J, Lu JS, Liu GY, Shen ZZ and Shao ZM. Outcome of pure mucinous breast carcinoma compared to infiltrating ductal carcinoma: a population-based study from China. Annals of surgical oncology. 2012; 19:3019-3027.

3. Toikkanen $\mathrm{S}$ and Kujari H. Pure and mixed mucinous carcinomas of the breast: a clinicopathologic analysis of 61 cases with long-term follow-up. Human pathology. 1989; 20:758-764.

4. Andre S, Cunha F, Bernardo M, Meneses e Sousa J, Cortez F and Soares J. Mucinous carcinoma of the breast: a pathologic study of 82 cases. Journal of surgical oncology. 1995; 58:162-167.

5. Zhang M, Teng XD, Guo XX, Zhao JS and Li ZG. Clinicopathological characteristics and prognosis of mucinous breast carcinoma. Journal of cancer research and clinical oncology. 2014; 140:265-269.

6. Tavassoli FA DP, editors. Pathology and genetics of tumours of the breast and female genital organs. Lyon: IARC Press; 2003. World Health Organization Classification of Tumours.

7. Lakhani SR, Ellis LO, Schnitt SJ, Tan PH, Van de Vijver MJ. WHO Classification of Tumours of the Breast. Fourth Edition. IARC: Lyon, 2012.

8. Komaki K, Sakamoto G, Sugano H, Morimoto T and Monden Y. Mucinous carcinoma of the breast in Japan. A prognostic analysis based on morphologic features. Cancer. 
1988; 61:989-996.

9. Rasmussen BB, Rose C and Christensen IB. Prognostic factors in primary mucinous breast carcinoma. American journal of clinical pathology. 1987; 87:155-160.

10. Norris HJ and Taylor HB. Prognosis Of Mucinous (Gelatinous) Carcinoma Of the Breast. Cancer. 1965; $18: 879-885$

11. Lacroix-Triki M, Suarez PH, MacKay A, Lambros MB, Natrajan R, Savage K, Geyer FC, Weigelt B, Ashworth A and Reis-Filho JS. Mucinous carcinoma of the breast is genomically distinct from invasive ductal carcinomas of no special type. The Journal of pathology. 2010; 222:282-298.

12. Jambal P, Badtke MM, Harrell JC, Borges VF, Post MD, Sollender GE, Spillman MA, Horwitz KB and Jacobsen BM. Estrogen switches pure mucinous breast cancer to invasive lobular carcinoma with mucinous features. Breast cancer research and treatment. 2013; 137:431-448.

13. Chen DN, Song CG, Ouyang QW, Jiang YZ, Ye FG, Ma FJ, Luo RC and Shao ZM. Differences in breast cancer characteristics and outcomes between Caucasian and Chinese women in the US. Oncotarget. 2015; 6:1277412782. doi: 10.18632/oncotarget.3666.

14. Fan L, Strasser-Weippl K, Li JJ, St Louis J, Finkelstein DM, Yu KD, Chen WQ, Shao ZM and Goss PE. Breast cancer in China. The Lancet Oncology. 2014; 15:e279-289.

15. Li J, Zhang BN, Fan JH, Pang Y, Zhang P, Wang SL, Zheng S, Zhang B, Yang HJ, Xie XM, Tang ZH, Li H, Li JY, He JJ and Qiao YL. A nation-wide multicenter 10-year (19992008) retrospective clinical epidemiological study of female breast cancer in China. BMC cancer. 2011; 11:364.

16. Zhang L, Jia N, Han L, Yang L, Xu W and Chen W. Comparative analysis of imaging and pathology features of mucinous carcinoma of the breast. Clinical breast cancer. 2015; 15:e147-154.

17. Weigelt B, Horlings HM, Kreike B, Hayes MM, Hauptmann M, Wessels LF, de Jong D, Van de Vijver MJ, Van't Veer LJ and Peterse JL. Refinement of breast cancer classification by molecular characterization of histological special types. The Journal of pathology. 2008; 216:141-150.

18. Toikkanen S, Eerola E and Ekfors TO. Pure and mixed mucinous breast carcinomas: DNA stemline and prognosis. Journal of clinical pathology. 1988; 41:300-303.
19. Matsukita S, Nomoto M, Kitajima S, Tanaka S, Goto M, Irimura T, Kim YS, Sato E and Yonezawa S. Expression of mucins (MUC1, MUC2, MUC5AC and MUC6) in mucinous carcinoma of the breast: comparison with invasive ductal carcinoma. Histopathology. 2003; 42:26-36.

20. Park S, Koo J, Kim JH, Yang WI, Park BW and Lee KS. Clinicopathological characteristics of mucinous carcinoma of the breast in Korea: comparison with invasive ductal carcinoma-not otherwise specified. Journal of Korean medical science. 2010; 25:361-368.

21. Bae SY, Choi MY, Cho DH, Lee JE, Nam SJ and Yang $\mathrm{JH}$. Mucinous carcinoma of the breast in comparison with invasive ductal carcinoma: clinicopathologic characteristics and prognosis. Journal of breast cancer. 2011; 14:308-313.

22. Chen L, Fan Y, Lang RG, Guo XJ, Sun YL, Cui LF, Liu FF, Wei J, Zhang XM and Fu L. Breast carcinoma with micropapillary features: clinicopathologic study and longterm follow-up of 100 cases. International journal of surgical pathology. 2008; 16:155-163.

23. Liu F, Yang M, Li Z, Guo X, Lin Y, Lang R, Shen B, Pringle $\mathrm{G}$, Zhang $\mathrm{X}$ and $\mathrm{Fu} \mathrm{L}$. Invasive micropapillary mucinous carcinoma of the breast is associated with poor prognosis. Breast cancer research and treatment. 2015; 151:443-451.

24. Shet $\mathrm{T}$ and Chinoy R. Presence of a micropapillary pattern in mucinous carcinomas of the breast and its impact on the clinical behavior. The breast journal. 2008; 14:412-420.

25. $\mathrm{Ng} \mathrm{WK}$. Fine-needle aspiration cytology findings of an uncommon micropapillary variant of pure mucinous carcinoma of the breast: review of patients over an 8-year period. Cancer. 2002; 96:280-288.

26. Komenaka IK, El-Tamer MB, Troxel A, Hamele-Bena D, Joseph KA, Horowitz E, Ditkoff BA and Schnabel FR. Pure mucinous carcinoma of the breast. American journal of surgery. 2004; 187:528-532.

27. Tseng HS, Lin C, Chan SE, Chien SY, Kuo SJ, Chen ST, Chang TW and Chen DR. Pure mucinous carcinoma of the breast: clinicopathologic characteristics and long-term outcome among Taiwanese women. World journal of surgical oncology. 2013; 11:139. 